\title{
Numerical Analysis of Film Cooling Effectiveness under Variable Blowing Ratio and Injection Angles
}

\author{
Mohammed Al-Hemyari, Mohammad O. Hamdan, Mehmet F. Orhan \\ Mechancial Engineering Department, American University of Sharjah \\ Sharjah, UAE \\ b00071156@aus.edu
}

\begin{abstract}
Higher allowable gas turbine inlet temperatures are limited by the maximum allowable temperature the turbine blade material can handle. In gas turbines using a cooling technique is crucial to allow higher gas turbine inlet temperature and to enhance the durability of the gas turbine blade. A higher gas turbine inlet temperature allows boosting gas turbine efficiency which reduces fuel consumption. Film cooling where a relatively low temperature air is used to form a protective layer of cool air around the blade to shield it from high temperature gases. Many complex interrelated geometry and flow parameters affect the effectiveness of the film cooling. The complex interrelations between these parameters are considered the main challenge in properly understanding the effect of these parameters on film cooling. Numerical techniques are viable analysis techniques that are used to improve film cooling techniques. In this study, a simplified 2D film cooling jet blown from a slot jet is used to understand the interconnected relation between blowing ratio and injection angle. The effect of injection angles on adiabatic film effectiveness was found to be negligible at very low blowing ratios. However, as the blowing ratio increases the effect of angle becomes pronounced. In general, for wide range of blowing ratio $\mathrm{M}=0.1-1.0$, decreasing blowing angle causes an increase in adiabatic film efficiency which mean film attachment is dominating the turbine blade shielding. But at high blowing ratio such as $\mathrm{M}=2$, the cool film thickness dominates the heat transfer hence high angle mean thicker cooling film. Increasing blowing angle to obtain thicker film eventually cause detachment of cooling film and deteriorate the adiabatic film effectiveness.
\end{abstract}

Keywords: CFD, Film Cooling, Gas Turbine.

\section{Nomenclature}

$2 \mathrm{D}$

$C_{p}$

$D$

EWF

$k$

$k_{t}$

$M$

$P$

RNG

$T$

$V$

VR

Greek symbols

\section{$\alpha$}

$\eta \quad$ film cooling effectiveness

$\mu \quad$ Dynamic viscosity, $\left[\frac{\mathrm{kg}}{\mathrm{m} \cdot \mathrm{s}}\right]$

$\rho \quad$ density

$\omega \quad$ turbulence specific dissipation rate turbulence dissipation rate

two-dimensional

Specific heat, $\left[\frac{\mathrm{J}}{\mathrm{kg} \cdot \mathrm{K}}\right]$

slot diameter, $[\mathrm{m}]$

enhanced wall function

turbulence kinetic energy, $\left[\mathrm{m}^{2} / \mathrm{s}^{2}\right]$

thermal conductivity, $\left[\frac{W}{m \cdot K}\right]$

blowing ratio

pressure, $[\mathrm{kPa}]$

Reynolds normalization group

temperature $[K]$

velocity $[\mathrm{m} / \mathrm{s}]$

velocity ratio of coolant to mainstream gas

injection angle 


$\begin{array}{ll}\text { Subscripts } & \\ \text { ad } & \text { adiabatic } \\ c & \text { coolant } \\ h & \text { mainstream } \\ \text { w } & \text { wall }\end{array}$

\section{Introduction}

A Gas turbine uses a high temperature mixture of air and fuel to spin turbine blades. The higher the inlet temperature at the turbine blade, the higher is the thermal efficiency of the gas turbine. However, working at higher temperatures affects the durability of a gas turbine [1]. To allow higher inlet temperature researchers to have done enormous work in the field of material science and cooling techniques. Different cooling techniques such as internal jet impingement [2-6] and film cooling [7-16] are used to maintain blade temperature below the material design constraint. In film cooling technique a relatively low temperature air is taken from the compressor is to flow through small holes over the turbine blade surface forming a protective layer on the turbine blade separating it from the hot mainstream gas [7]. This protective film layers reduces the heat transfer significantly between the hot mainstream gas and the turbine blade surface which allows higher mainstream gas to enter the turbine without negatively affecting the turbine durability [8].

The film cooling performance is reported using adiabatic film effectiveness which is defined as follow.

$$
\eta=\frac{T_{c}-T_{w a l l}}{T_{h}-T_{c}}
$$

Parameters affecting adiabatic film cooling effectiveness are interrelated. The main parameters are blowing ratio (M), injection angle $(\alpha)$ and density ratio (DR). Experimental and numerical work have showed that blowing ratio and injection angle are much interconnected. Blowing ratio is flux ratio between the coolant and the hot mainstream flow and can be expressed by combining the density and velocity of both coolant and hot mainstream as seen in equation (2)

$$
M=\frac{\rho_{c} V_{c}}{\rho_{h} V_{h}}
$$

Researchers have also tried to determine the optimum injection angle that gives the highest effectiveness under different conditions. Jia et al. [9] carried out a very wide experimental and numerical work for angles ranging between 16 and 90 and a wide range of blowing ratio ranging between 0.5 and 9 . They concluded that at the different values of blowing ratios, an injection angle of 30 achieved the highest film cooling effectiveness. Yuen et al. [10] experimentally investigated the interconnected relation between blowing ratio and angle ratio and found that for every angle there is an optimum blowing ratio. This result was proven numerically by the Nijo et al. [11].

Numerical work on film cooling technique is mostly focused on selecting the best turbulence model to effectively estimate film cooling performance. Most of the published work employs Reynolds-Averaged Navier-Stokes equations in predicting film-cooling performance. Flat-plate configuration is a very efficient approximation to investigate the effect of different flow and geometrical parameters on film cooling performance. A very challenging task is to successfully be able to model film cooling boundary layer to be able to have a consistent and accurate approximation of the heat transfer close to the wall and to be able to predict film cooling efficiency. Ferguson et al. [12] Investigated the Standard k- $\varepsilon$ and Reynold stress models with standard wall formulation, non-equilibrium wall modelling and two-layer wall treatment and RNG k- $\varepsilon$ with standard wall function. They reported that the Standard k- $\varepsilon$ with the two-layer wall treatment have showed the most accurate approximation of film cooling performance in comparison to the other combinations. Later work by York et al. [13] showed that RNG k- $\varepsilon$ with two layers wall treatment have successfully predicated the production of turbulent kinetic energy which leads to a good agreement between experimental and numerical reported film cooling efficiency. RNG k- $\varepsilon$ with the two layers wall treatment was proven to show a good agreement with experimental data under different conditions 
and geometries by many researchers. [14] [15] [16]. In this study an analysis on film cooling performance on a flat plate surface under different blowing ratios and injection angles are investigated using ANSYS Fluent.

\section{Numerical Modeling}

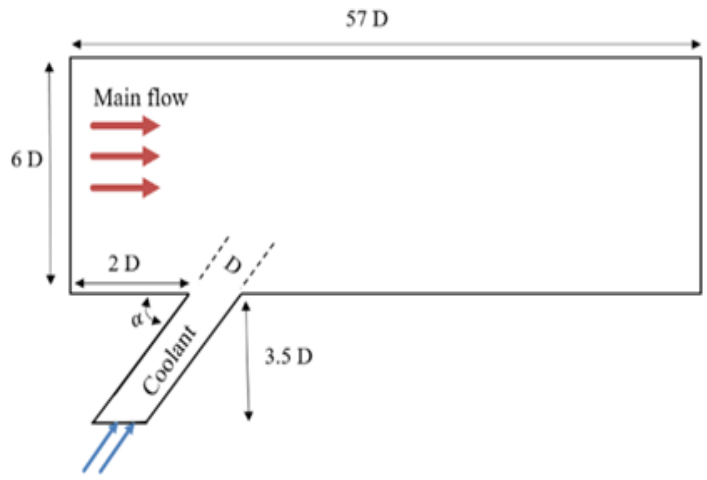

(a)

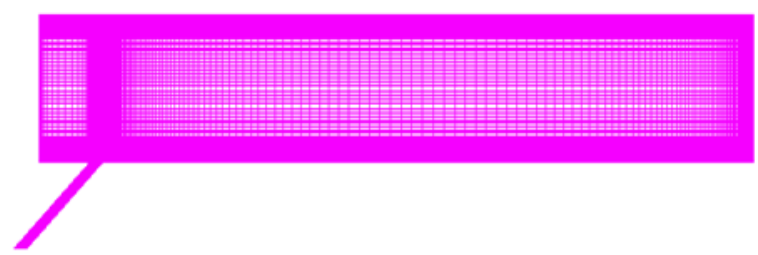

(b)

Fig. 1: Computational domain.

The schematic of the problem is show in Fig. 1, where the two inlets are referred two as main flow hot stream and jet coolant steam. The slot width is $\mathrm{D}, 40 \mathrm{~mm}$. The boundary layer length is $2 \mathrm{D}$, the coolant channel length is $3.5 \mathrm{D}$ and the length of the computational domain is 57D. The outlet is the far end of the mainstream flow direction. The dimensions follow the experimental work of O'Malley [17]. All the walls are nonslip and adiabatic. Enhanced wall treatment (EWF) is used in near-wall modelling for all K-e turbulence models. The outlet boundary is treated as pressure outlet with zerogauge pressure. The turbulent models standard k- $\varepsilon$, RNG k- $\varepsilon$, standard k- $\omega$, SST k- $\omega$ and SST models are used in this study.

Air is the working fluid for the mainstream and coolant jet. Air is treated as an incompressible ideal gas. Air density is calculated using the ideal gas equation were the air specific heat, thermal conductivity and dynamic viscosity are obtained from Eqs. 3-5 using the data given by Turns respectively where $\mathrm{T}$ is the temperature in kelvins.

Specific heat, $\mathrm{C}_{\mathrm{p}}(\mathrm{J} / \mathrm{kg} . \mathrm{K})$ :

$$
C_{p}=9.0813 * 10^{-11} \mathrm{~T}^{4}-4.8066 * 10^{-7} \mathrm{~T}^{3}+8.0735 * 10^{-4} \mathrm{~T}^{2}-0.32136 \mathrm{~T}+1.045 * 10^{3}
$$

Thermal conductivity, $\mathrm{k}(\mathrm{W} / \mathrm{m} . \mathrm{k})$ :

$$
k_{t}=7.995 * 10^{-12} \mathrm{~T}^{3}-2.4013 * 10^{-8} \mathrm{~T}^{2}+8.3047 * 10^{-5} \mathrm{~T}+2.8822 * 10^{-3}
$$

Dynamic viscosity, $\mu\left[\frac{\mathrm{kg}}{\mathrm{m} \cdot \mathrm{s}}\right]$ :

$$
\mu=1.7020 * 10^{-14} \mathrm{~T}^{3}-4.0405 * 10^{-11} \mathrm{~T}^{2}+6.8539 * 10^{-8} \mathrm{~T}+1.0616 * 10^{-6}
$$

A non-uniform structured mesh is generated using the commercial software pointwise. Since cells close to the wall are required to have a $\mathrm{y}^{+}$of 1.0 by the EWF treatment, cells close to the wall were given special treatment such that the height of the first cell closest to the wall was calculated to be 0.000170D. This was enough to have a $\mathrm{y}^{+}$value less than unity. Fig. 1 shows the generated mesh.

\subsection{Equations}

The governed equations to the steady 2-D flow are continuity conservation of momentum and conservation of energy: 


$$
\begin{gathered}
\frac{\partial\left(\rho \mathrm{u}_{\mathrm{i}}\right)}{\partial \mathrm{x}_{\mathrm{i}}}=0 \\
\frac{\partial\left(\rho \mathrm{u}_{\mathrm{j}} \mathrm{u}_{\mathrm{i}}\right)}{\partial \mathrm{x}_{\mathrm{j}}}=\frac{\partial \mathrm{P}}{\partial \mathrm{x}_{\mathrm{j}}}+\frac{\partial}{\partial \mathrm{x}_{\mathrm{j}}}\left[\mu\left(\frac{\partial \mathrm{u}_{\mathrm{i}}}{\partial \mathrm{x}_{\mathrm{j}}}+\frac{\partial \mathrm{u}_{\mathrm{j}}}{\partial \mathrm{x}_{\mathrm{i}}}\right)-\rho \overline{\mathrm{u}_{\mathrm{i}} \mathrm{u}_{\mathrm{j}}}\right] \\
\frac{\partial\left(\rho \mathrm{u}_{\mathrm{j}} \mathrm{T}\right)}{\partial \mathrm{x}_{\mathrm{j}}}=\frac{\partial}{\partial \mathrm{x}_{\mathrm{j}}}\left[\frac{\mu}{\operatorname{Pr}}\left(\frac{\partial \mathrm{T}}{\partial \mathrm{x}_{\mathrm{j}}}\right)-\rho \overline{\mathrm{T}}_{\mathrm{i}} \mathrm{u}_{\mathrm{j}}\right]
\end{gathered}
$$

Where $u_{i}$ is the mean velocity, $\mathrm{T}$ is the temperature and $\mathrm{P}$ is the pressure. The acute represent the fluctuations in the flow variable. The time averaged component in the momentum and energy equations are modelled using the Boussinesq hypothesis and the simple eddy diffusivity model as shown in the following equations respectively:

$$
\begin{gathered}
\rho \overline{u_{1} \hat{u}_{j}}=\mu_{t}\left(\frac{\partial u_{i}}{\partial x_{j}}+\frac{\partial u_{j}}{\partial x_{i}}\right)-\frac{2}{3} \rho k \delta_{i j} \\
\rho \overline{T_{1} \dot{u}_{j}}=-\frac{\mu_{t}}{\operatorname{Pr}_{t}}\left(\frac{\partial T}{\partial x_{j}}\right)
\end{gathered}
$$

Where $\mu_{\mathrm{t}}$ is the turbulent viscosity, $\mathrm{k}$ is the turbulent kinetic energy and $\operatorname{Pr}_{\mathrm{t}}$ is the turbulent Prandtl number. To solve equations 12 and 13 the difference turbulence models are used to estimate the different turbulent parameters that appears in equations 14 and 15.

In this work the governing equations were discretized using the second-order upwind scheme and the pressure-velocity coupling was resolved using the semi implicit method for pressure linked equations corrected (SIMPLEC). Solution convergent is determined when the energy residual was lower than $10^{-9}$ and the other equations lower than $10^{-6}$.

Table 1: Parameters used in the numerical model.

\begin{tabular}{|l|l|l|l|l|l|l|}
\hline Run & $\alpha$ & $\mathrm{V}_{\mathrm{c}}$ & $\mathrm{V}_{m s}$ & $\mathrm{M}$ & $T_{\mathrm{c}}(K)$ & $T_{m s}(K)$ \\
\hline Validation & 90 & 2.3 & 23 & 0.13 & 300 & 373 \\
\hline Case\#1 & $30,60,90$ & 2.5 & 30 & 0.1 & 300 & 360 \\
\hline Case\#2 & $30,60,90$ & 25 & 30 & 1 & 300 & 360 \\
\hline Case\#3 & $30,60,90$ & 50 & 30 & 2 & 300 & 360 \\
\hline
\end{tabular}

\subsection{Validation and Mesh Independence}

This work was validated using the numerical work of Bayraktar [14]. In his work the adiabatic film effectivenss was reported for a combination of flow parameters for slot film cooling. Same flow parameters were used as mentioned in Table 1 to validate the model. 


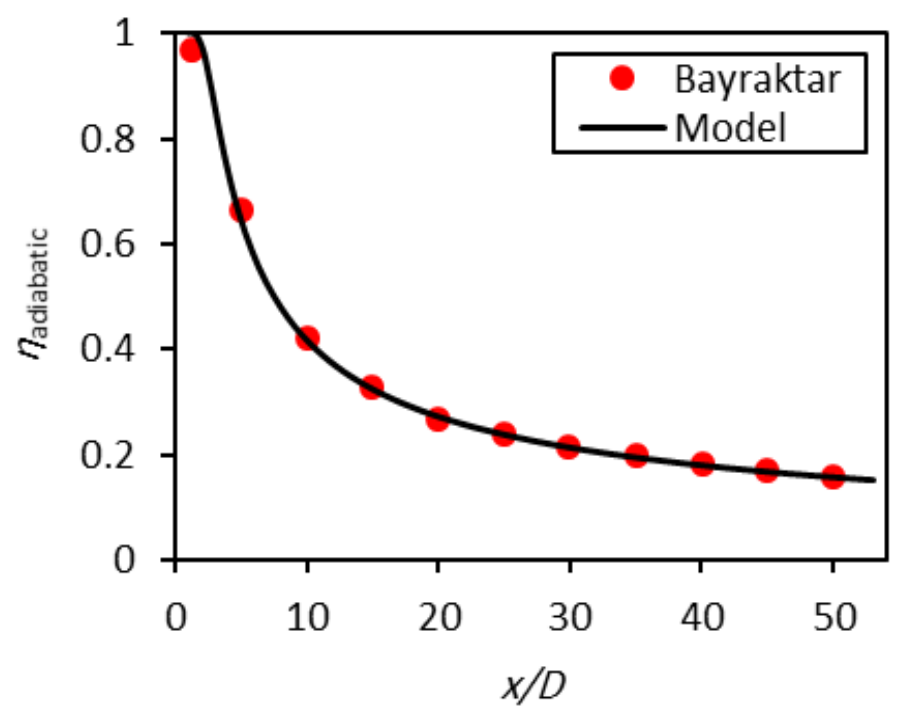

Fig. 2: Numerical validation of the model using adiabatic film effectivenss .

The numerical validation against Bayraktar [14] numerical analysis using the flow parameters in Table 1 is shown in Fig. 2. The figure shows a good agreement of the numerical results against the published work. The error was calculated to be less than $1 \%$.

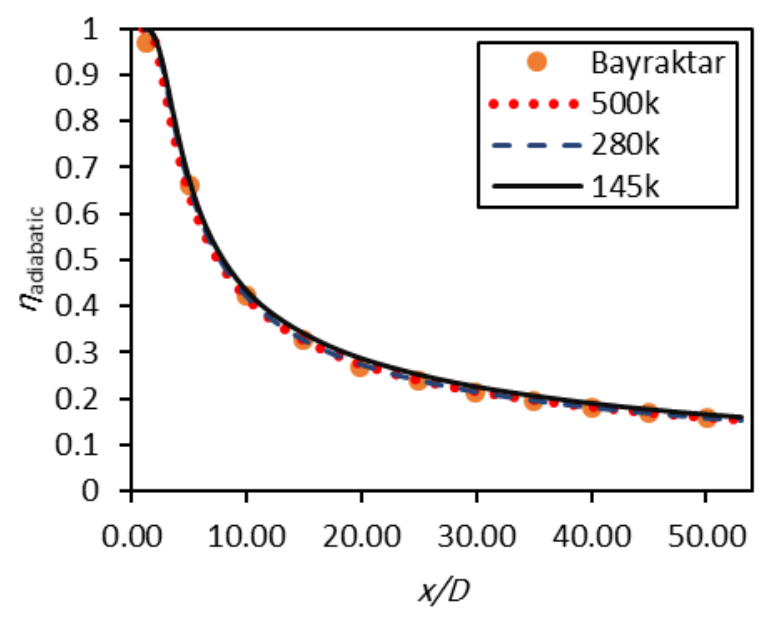

(a) Mesh independent study

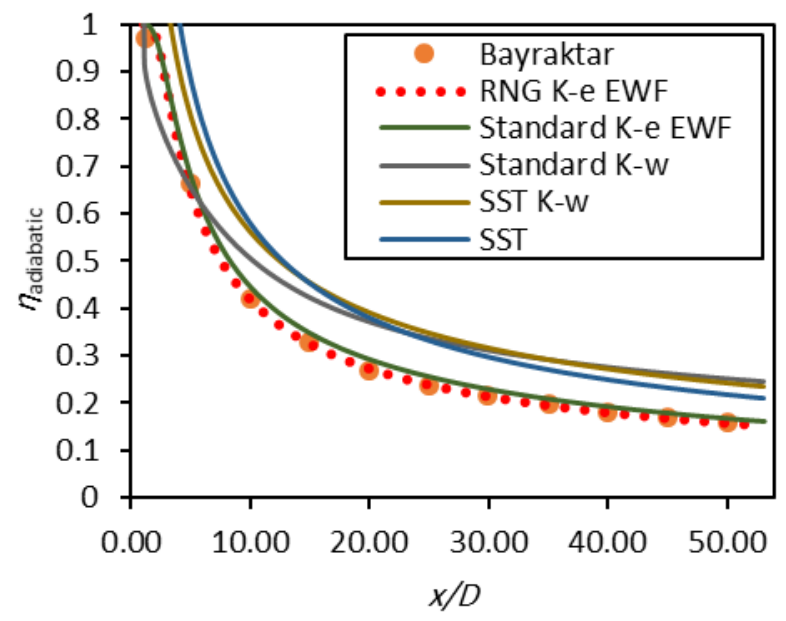

(b) Turbulence model testing

Fig. 3: Mesh independence study and turbulence model selection.

The mesh independent study can be seen in Fig. 3-a using three different mesh sizes; 500k, 280k and 145k elements. The figure shows that a noticeable error is seen when a $145 \mathrm{k}$ mesh element size is used (3\%). Therefore, a $280 \mathrm{k}$ element size were chosen to test the different turbulence models seen in Fig. 3-b. The different k- $\varepsilon$ turbulence models have shown that they are best in predicting the adiabatic film effectiveness as where RNG k- $\varepsilon$ turbulence model have shown the best agreement with numerical work. 


\section{Results}

The adiabatic film effectiveness is used to report the performance of film cooling under different parameters. Equation 1 can be re-written into equation 11 , where adiabatic condition is refereed to using a subscript, ad.

$$
\eta_{a d}=\frac{T_{h o t}-T_{a d-w a l l}}{T_{h o t}-T_{\text {cold }}}
$$

A high adiabatic film effectiveness is achieved when the coolant is effectively protecting the turbine blade from the hot mainstream. While a low adiabatic film effectiveness is when the protective layer is not effectively protecting the turbine blade. A low adiabatic film effectiveness means that the cool film is not effective in shielding the wall from the hot mainstream flow. While a high adiabatic film effectiveness means that the cool film is effective in shielding the wall from the hot mainstream flow. As the flow is moving the mainstream flow direction the wall temperature will rise due to heat transfer to the wall causing the adiabatic film effectiveness to drop. This can be seen clearly in Fig. 4 (a,b and c).

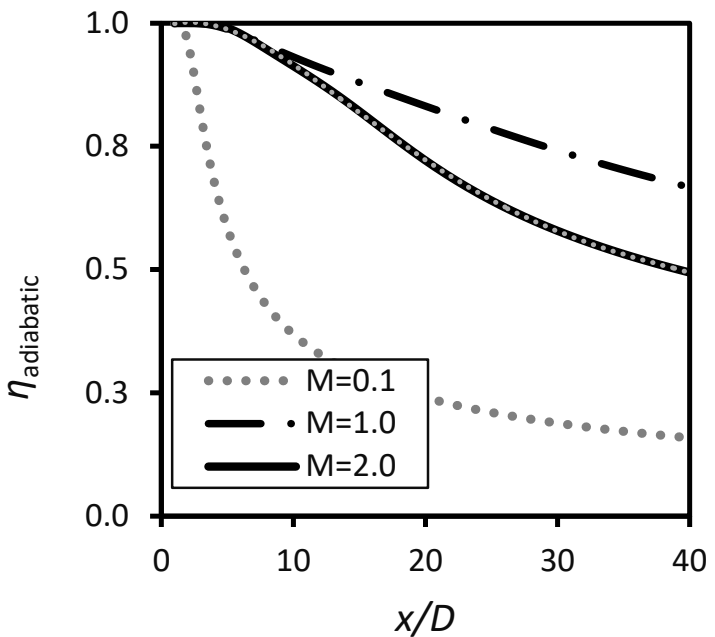

(a) $\alpha=30$

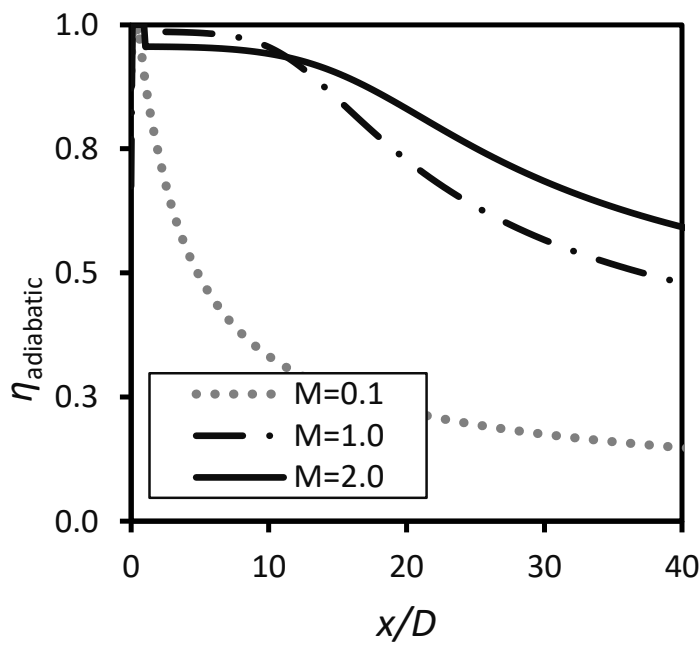

(c) $\alpha=90$

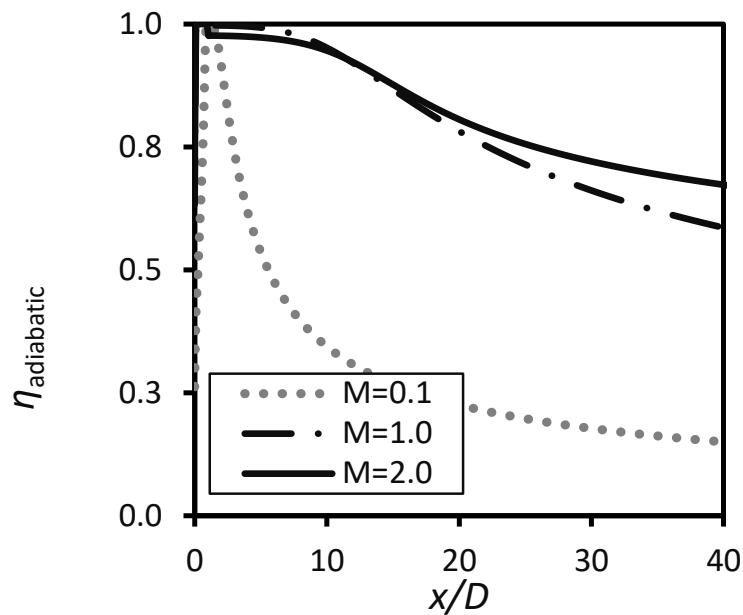

(b) $\alpha=60$

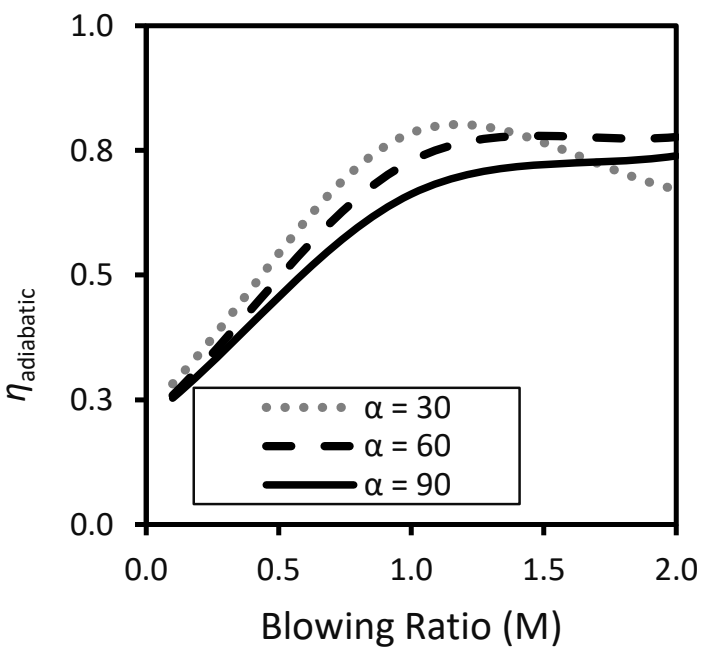

(d) averaged adiabatic film effectiveness at different blowing ratios for all angles

Fig. 4: Numerical results for all the cases in Table 1. 
Adiabatic film effectiveness depends on the size of the protection layer and how far this protective layer is attached to the turbine blades. A small blowing ratio means a low chance of fluid detachment but also it means thin protective layer of cool fluid. A high blowing anlage means a high chance of detachment but also it means a thick protective layer of cool fluid. These contradicting effects depends of blowing ratio, makes the adiabatic film effectiveness sensitive to blowing angle. Similarly, A small blowing angle means a low chance of fluid detachment but also it means thin protective layer of cool fluid. A high blowing angle means a high chance of detachment but also it means a thick protective layer of cool fluid. This very complex interrelated relation between theses parameters is show in Fig. 4-d. For the studied parameters the highest achieved adiabatic film effectiveness is achieved using a blowing ratio $\mathrm{M}=0.1$ and an injection angle of $\alpha=30$.

As shown also in Fig. 4-a for blowing angle of 30 degree, the adiabatic film effectiveness has non-monotonic relation with blowing ratio. While as shown Fig. 4-b and Fig. 4-c for blowing angle of 60 and 90 degree, a monotonic relation exists between the adiabatic film effectiveness and the blowing ratio. The increase in film efficiency with blowing ratio is expected since more fluid discharges from the film hole and bigger protective layer of cool air is formed between the hot mainstream flow and the surface causing better surface shielding. However, this trend is not indefinite since as discharging increases beyond $M=1.0$ (for blowing angel of 30 degree), the chance of film detachment from the surfaces increases causing improper protection and less effectiveness of forming a cool film to shield the surface from the hot mainstream. The decrease in adiabatic film effectiveness means that more heat is transferred to the turbine blade causing it is temperature to rise.

\section{Conclusion}

The effect of blowing angle is negligible at low blowing ratio $(\sim \mathrm{M}=0.1)$ which mean the injected flow has little effect to no-effect on forming the film blanket which will be mainly dominated by the mainstream flow boundary layer. A low blowing ration means the flow has low momentum and energy to affect the mainstream flow which reduces the effect of blowing angle. However once blowing ratio increases beyond $\mathrm{M}=0.1$, the blowing angle become an important factor. The effect of blowing ratio is complex since it relates quantitatively the effect of flow linear momentum of two streams without counting the effect of direction of these quantities. Linear momentum is a vector parameter and its impact on the flow is determine by the quantitative value and the flow vector direction. For blowing ratio equal 1 or less, as shown in Fig. 4, the adiabatic film effectiveness increases as the blowing angel is decreases. The smaller the angle the higher the chance that the flow remain in contact with the surface forming a cool blanket that shield the surface from the hot mainstream. However once high blowing ratio is used the angle effect become less important and the previous relation fails. This behaviour is explained that as blowing ratio increases, more mass of cool fluid become available to shield the surface from the hot mainstream.

In general, for wide range of blowing ratio $M=0.1-1.0$, decreasing blowing angle causes an increase adiabatic film efficiency which mean film attachment is dominating the turbine blade shielding. But at high blowing ratio such as $\mathrm{M}=2$, the cool film thickness dominates the heat transfer hence high angle mean thicker cooling film. Increasing blowing angle to obtain thicker film eventually cause detachment of cooling film and deteriorate the adiabatic film effectiveness.

\section{References}

[1] M. R. Reyhani, M. Alizadeh, A. Fathi and H. Khaledi, "Turbine blade temperature calculation and life estimation - a sensitivity analysis," Propulsion and Power Research, vol. 2, no. 2, pp. 148-161, 2013.

[2] E. Elnajjar, M. Hamdan and Y. Haik, "Experimental Investigation of Internal Channel Cooling Via Jet Impingement," FDMP: Fluid Dynamics \& Materials Processing, vol. 9, no. 1, pp. 77-89, 2013.

[3] M. Hamdan and A. Hayek, "Effect of Cavity Size on Confined Slot Jet Impingement Cooling," in ASME 2013 Heat Transfer Summer Conference, Minneapolis, 2013.

[4] M. Hamdan, E. Elnajjar and Y. Haik, "Measurement and Modeling of Confined Jet Discharged Tangentially on a Concave Semicylindrical Hot Surface," Journal of Heat Transfer-transactions of The Asme, vol. 133, no. 12, p. 122203, 2011.

[5] M. Hamdan and M. Al-Nimr, "Thermal Augmentation in Internal Cooling Passage by Converting Impingement Jet to Induced Swirl Flow," in 6th International Conference on Computational Heat and Mass Transfer, Guangzhou, 
2009.

[6] E. Elnajjar, M. Hamdan and Y. Haik, "Experimental Investigation of Impinging Jet Flow on a Heated Curved Surface," in 6th International Conference on Thermal Engineering, Istanbul, 2012.

[7] D. Bogard, "Gas Turbine Film Cooling," Journal of Propulsion and Power, 2005.

[8] R. J. Goldstein, "Film Cooling," Advances in Heat Transfer, vol. 7, pp. 321-379, 1971.

[9] R. Jia, B. Sundén, P. Miron and B. Léger, "A Numerical and Experimental Investigation of the Slot Film-Cooling Jet With Various Angles,” Journal of Turbomachinery, vol. 127, no. 3, pp. 635-645, 2005.

[10] C.H.N.Yuen and R.F.Martinez-Botas, "Film cooling characteristics of a single round hole at various streamwise angles in a crossflow: Part I effectiveness," International Journal of Heat and Mass Transfer, vol. 46, no. 2, pp. 221-235, 2003.

[11] N. Jose, J. J.S and G. B. Yepuri, "Numerical Investigation of Adiabatic Film Cooling Effectiveness over a Flat Plate Model With Cylindrical Holes," in International Conference on Computational Heat and Mass Transfer , 2015.

[12] J. D. Ferguson, D. K. Walters and J. H. Leylek, "Performance of Turbulence Models and Near-Wall Treatments in Discrete Jet Film Cooling Simulations," in ASME 1998 International Gas Turbine and Aeroengine Congress and Exhibition, Stockholm, 1998.

[13] W. D. York and J. H. Leylek, "Leading-Edge Film-Cooling Physics: Part I - Adiabatic Effectiveness," in ASME Turbo Expo 2002: Power for Land, Sea, and Air, Amsterdam, 2002.

[14] S. Bayraktar and T. Yilmaz, "Two-dimensional numerical investigation of film cooling by a cool jet injected at various angles for different blowing ratios," J. Mechanical Engineering Science, vol. 222, no. c, pp. 1215-1224, 2008.

[15] K. Singh, B. Premachandran and M. R. Ravi, "A Numerical Study on the 2D Film Cooling of a Flat Surface," Numerical Heat Transfer, vol. 67, pp. 673-695, 2014.

[16] C. Q. Nguyen, N. V. T. Tran, B. C. Bernier, S. H. Ho and J. S. Kapat, "Sensitivity Analysis for Film Effectiveness on a Round Film Hole Embedded in a Trench Using Conjugate Heat Transfer Numerical Model," in ASME Turbo Expo 2010: Power for Land, Sea, and Air, Glasgow, 2010.

[17] K. O'Malley, Theoritcal aspects of film cooling, PhD Thesis, University of Oxford, 1984. 